Canadian

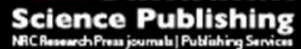

Canadian Journal of Physiology and Pharmacology Revue canadienne de physiologie et pharmacologie

\title{
MDCK cells are capable of water secretion and re-absorption in response to changes in the ionic environment
}

\begin{tabular}{|r|l|}
\hline Journal: & Canadian Journal of Physiology and Pharmacology \\
\hline Manuscript ID & cjpp-2016-0051.R2 \\
\hline Manuscript Type: & Article \\
\hline Date Submitted by the Author: & 08-Jul-2016 \\
\hline Complete List of Authors: & $\begin{array}{l}\text { Capra, Janne; Oulun Yliopisto Laaketieteellisen tiedekunta, Institute of } \\
\text { Diagnostics } \\
\text { Eskelinen, Sinikka; Oulun Yliopisto Laaketieteellisen tiedekunta, Institute of } \\
\text { Diagnostics }\end{array}$ \\
\hline Keyword: & $\begin{array}{l}\text { hyperpolarization, depolarization, 3D cell culture, spinning disc confocal } \\
\text { microscopy, kidney epithelial cells }\end{array}$ \\
\hline
\end{tabular}

SCHOLARONE $^{\text {sm }}$
Manuscripts 
MDCK cells are capable of water secretion and re-absorption in response to changes in the ionic environment

Capra, Janne $\mathrm{P}^{1}$; Eskelinen ${ }^{1 *}$, Sinikka M

${ }^{1}$ Biocenter Oulu and the Institute of Diagnostics, University of Oulu, Oulu, Finland

Correspondence to: sinikka.eskelinen@oulu.fi 


\section{Abstract}

A prerequisite for tissue electrolyte homeostasis is highly regulated ion and water transport through kidney or intestinal epithelia. In the present work, we have monitored changes in the cell and luminal volumes of type II Madin-Darby canine kidney (MDCK) cells grown in a 3D environment in response to drugs, or to changes in the composition of the basal extracellular fluid. Using fluorescent markers and high-resolution spinning disc confocal microscopy, we could show that lack of sodium and potassium ions in the basal fluid (tetramethylammonium chloride (TMACl) buffer) induces a rapid increase in the cell and luminal volumes. This transepithelial water flow could be regulated by inhibitors and agonists of chloride channels. Hence, the driving force for the transepithelial water flow is chloride secretion, stimulated by hyperpolarization. Chloride ion depletion of the basal fluid (using sodium gluconate buffer) induces a strong reduction in the lumen size, indicating re-absorption of water from the lumen to the basal side. Lumen size also decreased following depolarization of the cell interior by rendering the membrane permeable to potassium. Hence, MDCK cells are capable of both absorption and secretion of chloride ions and water; negative potential within the lumen supports secretion, while depolarizing conditions promote re-absorption.

Keywords:

- hyperpolarization

- depolarization

- 3D cell culture

- spinning disc confocal microscopy

- transepithelial water transport

- chloride secretion

- kidney epithelial cells 


\section{Introduction}

The functionality of both secretory and absorptive organs is dependent upon the lumen size and transepithelial transport capacity, which requires an appropriate segregation of transporters and channels to the apical and basal domains (Datta et al. 2011; Rodriguez-Boulan and Macara 2014). A characteristic of mammalian kidney function is glomerular filtration and the subsequent reabsorption of sodium and chloride ions and water in the renal tubules. Glomerular filtration may be reduced, or even abolished, during renal failure, leaving only tubular secretory mechanisms in the nephrons as a means to form urine. Efficient secretory and absorptive mechanisms are especially needed when the organism is faced with drastic changes in the environment. In order to remain functional under these conditions, the kidney epithelium must have the capacity to regulate transcellular transport, as well as the cell and luminal volumes (Grantham and Wallace 2002).

Fluid secretion through epithelial cells is initiated by chloride secretion. Chloride channels can be classified according to their gating characteristics: (i) extracellular ligand-gated channels (GABA and glycine receptors), (ii) voltage-gated chloride channels (ClC-family), (iii) cAMP-PKA activated channel (cystic fibrosis transmembrane conductance regulator CFTR), (iv) volume-regulated, or volume-sensitive anion channels (VRAC, VSOR, VSOAC, VSORCC), and (v) calcium-activated chloride channels (CaCCs) (Hoffmann et al. 2014). Some of these, such as the ClC family and CaCCs (anoctamins) are widely expressed, whereas CFTR, stimulated by CAMP, is expressed in epithelia and cell lines derived from secretory organs (Bi et al. 2014; Kunzelmann 2015; Tanner et al. 1992; Terryn et al. 2011; Wallace 2011). CFTR-induced water transport is especially evident in autosomal dominant polycystic kidney disease (ADPKD), the most frequent inherited nephropathy; the disease is characterized by liquid-filled cysts that ultimately occupy the whole kidney (Sullivan et al. 1998; Terryn et al. 2011; Wallace 2011). A widely accepted model of cAMP-mediated cyst expansion consists of apical chloride ion secretion through CFTR and calcium-activated chloride channel anoctamin 1 (ANO1, also called TMEM 16), compensated by basal chloride ion influx via $\mathrm{Na}^{+}-\mathrm{K}^{+}-2 \mathrm{Cl}^{-}$ cotransporters (Buchholz et al. 2014a; Tanner et al. 1992). Increased apical $\mathrm{Cl}^{-}$and basal $\mathrm{K}^{+}$conductance 
creates a negative transepithelial potential in the lumen, which is balanced by the transport of sodium ions to the luminal side. The net addition of sodium and chloride ions into the luminal fluid drives the osmotic movement of water into the lumen (Buchholz et al. 2014a; Frizzell and Hanrahan 2012; Noda et al. 2010; Verkman et al. 2014; Wallace 2011).

Although the hormonal and chemical stimuli regulating the secretion and absorption of ions and water via epithelial pumps and channels are well characterized, very little is known about the actual mechanisms by which the cells respond structurally to these stimuli. Madin-Darby canine kidney (MDCK) cells form cysts when cultured in a collagen I or matrigel matrix. These cells have served as a kidney cell model for the analysis of the regulation of lumen formation and enlargement in response to chloride and water secretion in the presence of forskolin, which leads to elevation of cAMP (Buchholz et al. 2014a; Li et al. 2004; Tanner et al. 1992; Yang et al. 2008; Yuajit et al. 2013). In all of these experiments, the authors have used MDCK cells expressing endogeneous or transfected CFTR, and cyst enlargement occurred over culture periods of 6 to 12 days, during which time it can be reasonably assumed that cell proliferation and cell rearrangement both contributed significantly to the observed structural changes of the cyst and lumen.

There are several MDCK strains, of which at least nine seem to be unique (Dukes et al. 2011). The type I MDCK strain is isolated from a low passage parental cell line, whereas the type II strain is isolated from a high passage parental cell line. Type I MDCK cells originate from the distal tubule; they have a very high transepithelial resistance, very tight junctions and express CFTR. Type II MDCK cells are thought to originate from the proximal tubule; they lack CFTR expression (Mohamed et al. 1997), they are larger and taller than the rather flat type I cells, and they are the most commonly used MDCK strain in studies of epithelial polarity and lumen formation (Dukes et al. 2011).

In this work, we present a method allowing to analyse the effect of external stimuli on cell, lumen and cyst size over short periods of time and, hence, draw conclusions directly related to the ion and water transport capacity through the epithelium in response to changes in transepithelial potential, without interference from changes related to cell proliferation. We have chosen to use the type II MDCK cell strain, because it 
forms well-developed cell cysts; the cyst and luminal areas, and cell height can be measured and cyst, cell and lumen volumes are easily calculated on the basis of the regular spherical form of the type II MDCK cyst.

\section{Materials and methods}

Confocal spinning disc time-lapse microscopy was employed to monitor MDCK cystogenesis and cyst behaviour under various conditions with the aid of fluorescent markers. Imaging software was used to analyze the cyst shape and measure the changes in the lumen and cell volume, the apical and basal surface areas, and the cell height, i.e. the length of the apico-basal axis.

\section{Cell strains and culture conditions}

The type II MDCK cell line stably transfected with a vector with Venus-fluorophore tagged CD59 gene was a kind gift from Dr. Reika Watanabe and Dr. Guillaume Castillon (received $1^{\text {st }}$ of July, 2010)(Castillon et al. 2013; Rivier et al. 2010). Type II MDCK cells were originally purchased from the European Collection of Cell Cultures (Salisbury, United Kingdom; catalogue no. 00062107) (Castillon et al. 2013). The cells were grown in cell culture flasks at $37^{\circ} \mathrm{C}$ with Eagle's minimal essential medium with Earle's salts (E-MEM Sigma-Aldrich St. Louis, MO, USA). The medium was supplemented with $10 \%$ fetal bovine serum (FBS, HyClone, GE Healthcare, Little Chalfront, United Kingdom) and penicillin-streptomycin (100 U and $100 \mu \mathrm{g}$ per ml, Life Technologies, Carlsbad, CA, United States).

\section{Reagents and antibodies}

Hanks' Balanced Saline Solution (HBSS) was from Sigma-Aldrich. 1-(4-Trimethylammoniumphenyl)-6Phenyl-1,3,5-Hexatriene $p$-Toluenesulfonate (TMA-DPH) membrane dye and DAPI nuclear dye were from Life Technologies. Polyclonal anti-occludin rabbit antibody was from Zymed Laboratories Inc. (catalog number 71-1500) and was used at a 1:50 dilution. Monoclonal mouse antibody against canine E-cadherin (rr1) (Developmental Studies Hybridoma Bank, lowa City, IA, USA), was used at a 1:50 dilution. Polyclonal Texas Red goat anti-rabbit antibody (catalog number T2767), and polyclonal Alexa 488 chicken anti-mouse antibody (catalog number A-21200) were both purchased from Life Technologies and used at dilutions of 
1:100. The validation of the antibodies was based on the original references given by the suppliers. The antibodies were diluted in PBS containing $0.2 \mathrm{M}$ glycine and $5 \%$ FBS. Forskolin, nigericin, $\mathrm{CFTR}_{\text {inh }}-172$ (inhibitor of CFTR and volume-sensitive outwardly rectifying chloride conductance (VSORCC) channel, Melis et al. 2014) and lubiprostone (activator of CFTR and CIC-2 channel, Bijvelds et al. 2009; Cuppoletti et al. 2004) were purchased from Sigma-Aldrich. Calcium-activated chloride channel (ANO1) inhibitor, $\mathrm{CaCC}_{\text {inh- }}{ }^{-}$ A01 (Namkung et al. 2011), was purchased from Merck-Millipore (Billerica, MA, USA). EGTA (Sigma -Aldrich) was used at a final concentration of $1 \mathrm{mM}$ in PBS buffer $\left(\mathrm{w} / \mathrm{o} \mathrm{Ca}^{2+}\right)$ containing $18 \mathrm{mM}$ glucose.

\section{Spinning disc microscopy of living cells}

For 3D experiments, Matrigel (BD Biosciences, Franklin Lakes, NJ, USA) was used at a dilution of 1:1 in EMEM. 150000 cells were seeded on top of a solidified layer of Matrigel on $35 \mathrm{~mm}$ plate, as described earlier (Debnath et al. 2003; Töyli et al. 2010) and cells were allowed to grow for 3 to 5 days. To overcome the optical depth limitations imposed by the objectives of the microscope, and thus visualize whole cell cysts, $10-\mathrm{mm}$ round coverslips were placed at the bottom of the plate before the Matrigel was cast. When the time-lapse experiment was to be initiated, the cover slip was picked up, inverted and placed on a 35 $\mathrm{mm}$ glass-bottom plate, the Matrigel against the glass (Yagi et al. 2012), together with $500 \mu \mathrm{l} \mathrm{E-MEM}$ containing $10 \mu \mathrm{M} \mathrm{TMA}-\mathrm{DPH}$, and incubated at $+37^{\circ} \mathrm{C}$ for $30 \mathrm{~min}$ in a cell culture incubator. Before the experiments, the cover slip was washed three times with HBSS. $500 \mu$ l of TMACl buffer, $\mathrm{KCl}$ buffer with or without nigericin, or sodium gluconate buffer, were then added, and the cells were transferred to the incubation chamber of the microscope. 5-15 cysts were selected from each sample for time-lapse analysis and were visualized with a Zeiss Cell Observer spinning disc confocal microscope at 30-min intervals, at + 37 ${ }^{\circ} \mathrm{C}$, until they collapsed. A Z-stack of each cyst at each time point was collected. Excitation wavelengths of 405 and 488 nm, EC Plan NeoFluar 40x/0.75 DIC air objective, and BP 450/50nm (blue) and BP 525/50nm (green) emission filters were used. The experiments were repeated at least two times.

\section{Manipulation of membrane potential and activity of chloride transporters}


The TMACl, $\mathrm{KCl}$ and sodium gluconate buffers and the nigericin dilutions were prepared as described earlier by Vääräniemi and co-workers with the exception that sodium gluconate buffer contained $5.7 \mathrm{mM} \mathrm{CaCl} 2$ instead of $1 \mathrm{mM}$ in order to compensate for the $\mathrm{Ca}^{2+}$ buffering capacity of gluconate ions (Li et al. 2004; Vääräniemi et al. 1994; 1997). The buffer compositions are summarized in Table 1 and their osmolalities were as described by Vääräniemi et al. (1997).

Stock solutions of $10 \mathrm{mM}$ forskolin, $10 \mathrm{mM} \mathrm{CFTR}_{\text {inh }}-172,20 \mathrm{mM} \mathrm{CaCC} \mathrm{inh}_{\text {- }}-\mathrm{A} 01$ and $10 \mathrm{mM}$ lubiprostone were made in DMSO. $\mathrm{CFTR}_{\text {inh }}-172$ and $\mathrm{CaCC}_{\text {inh }}-\mathrm{A} 01$ were then diluted in TMACl buffer to make a $10 \mu \mathrm{M}$ and 20 $\mu \mathrm{M}$ working solutions, respectively. Lubiprostone was diluted in $\mathrm{HBSS}$ or $\mathrm{KCl}$ buffer to make a $10 \mu \mathrm{M}$ working solution. For experiments with forskolin, cells were grown in the presence of $10 \mu \mathrm{M}$ forskolin and fresh supplements were added to the cells daily. The total concentration of DMSO that the cells were exposed to was never higher than $0.1 \%(v / v)$. The effect of DMSO on cell and cyst area was evaluated and was found to have no detectable impact on cyst behavior (data not shown).

\section{Preparation of fixed samples for confocal microscopy}

For confocal microscopy of cell cysts, a 20 mm flexiPERM conB silicon adapter (Greiner Bio-One, Monroe, NC, USA) was attached to an objective slide and Matrigel-E-MEM solution was applied and allowed to solidify. 60000 cells were seeded on top of the Matrigel, as described by Debnath and co-workers (2003) and Töyli and co-workers (2010), and the cells were allowed to grow for 3-5 days. In order to visualize the integrity of tight junctions in the various media, the cells were then incubated in the presence of HBSS, $\mathrm{TMACl}$, or sodium gluconate, for $120 \mathrm{~min}$ at $+37^{\circ} \mathrm{C}$. For comparison, the cells were incubated for the same 120-min period in the presence of $1 \mathrm{mM} \mathrm{EGTA}$, a calcium chelator known to open cell-cell junctions.

The fixation method was as follows: after incubation in a given medium, the cysts within the Matrigel were washed three times with PBS, fixed with $500 \mu$ l of $1 \%$ paraformaldehyde (PFA) for $15 \mathrm{~min}$ at room temperature, and carefully washed three times for 10 min with PBS. $500 \mu$ of $0.2 \%$ Triton-X in PBS were added and the cysts were incubated at room temperature for $15 \mathrm{~min}$. The Triton- $\mathrm{X}$ solution was then replaced with $150 \mu$ l of primary antibody solution, and the cysts were incubated for $4 \mathrm{~h}$ at room 
temperature, and washed with PBS for $3 \times 10 \mathrm{~min}$. This procedure was repeated with the secondary antibodies, except the cysts were incubated for $1 \mathrm{~h}$ at room temperature. The cysts were then washed $3 \times 10$ min with PBS and twice with distilled water. After careful aspiration of the water, the silicon ring was removed and excess water was aspirated. Shandon Immu-Mount (Thermo Scientific, Waltham, MA, USA) was added, a cover glass $(24 \times 40 \mathrm{~mm})$ was placed on top of the cysts and the sample was allowed to dry over-night. Experiments were repeated 2-3 times.

\section{Confocal microscopy of fixed cells}

The Z-stack images of cysts were captured with an Olympus FluoView 1000 laser scanning confocal microscope using UPLSAPO 60x/1.35 oil-immersion objective. Excitation wavelengths of 405, 488 and 543 $\mathrm{nm}$ and appropriate emission filters were used for DAPI, rr1 and occludin, respectively.

\section{Quantification of lumen and cyst size}

For each condition visualized with the spinning disc microscope, 8-36 cysts were selected for quantitative analysis. For each time point, a layer from the $Z$-stack where the surface areas of the cyst and lumen were the largest was selected. This maximal cross-sectional area of the lumen and the cyst in the selected layer was measured with the Zen image processing program provided by Carl Zeiss AG. Mean values and standard errors were calculated and plotted onto a graph using Excel (Microsoft, Redmond, WA, USA). Imaris software (Bitplane, Belfast, United Kingdom) was used to render a three-dimensional representation of the luminal space, using the Surface function (Fig 1). Based on the assumption that both the cyst and lumen are spherical, the radii of the cyst and lumen were determined and their volumes and surface areas were calculated. The volume of the cells was obtained by subtracting lumen volume from cyst volume.

The number of cysts measured for each experiment was as follows: HBSS, $n=19$, two sets of experiments;

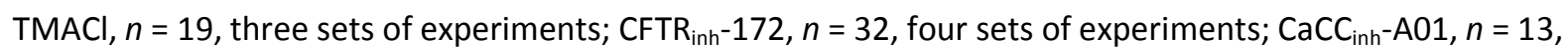
two sets of experiments; lubiprostone in HBSS, $n=28$, three sets of experiments; forskolin, $n=11$, two sets of experiments; forskolin + TMACl, $n=12$, two sets of experiments; Na-gluconate, $n=15$, two sets of 
experiments; $\mathrm{KCl}, n=17$, two sets of experiments; $\mathrm{KCl}$ with nigericin, $n=14$, two sets of experiments; lubiprostone in $\mathrm{KCl}, n=8$, two sets of experiments.

\section{Quantification of cell height}

To quantify the changes in cell height caused by the expanding lumen, a layer from the Z-stack in which the surface area of the lumen was the largest was selected for time points of 0 and 2.5 hours. For each such section, the cell height in eight directions was measured with the Zen image processing program (Carl Zeiss AG). The directions chosen were the same for every cyst. The ratios of the measurements taken at 2.5 hours relative to the 0 hour time point value were determined and the mean values were calculated from these ratios.

\section{Statistics}

Two-tailed Student's paired t-test with equal variance was used to assess the significance of the changes of lumen and cyst areas with time. $P$-values were categorized as not significant $(P>0.05)$, significant $(P<0.05$, denoted with $*)$ and very significant $(P<0.01$, denoted with $* *)$.

\section{Results}

In the present work, we have established a MDCK cell culture model for the analysis of the dynamics of lumen formation over short time-spans, and visualization of the cysts, cells and lumina using high resolution confocal microscopy and imaging software. The approach described here allows to minimize the contributions of cell number changes related to proliferation or apoptosis that inevitably occur in longer term culture settings, and that can significantly complicate the interpretation of the experimental observations.

We have employed the method to elucidate the cells' capacity to respond to changes in the composition of the extracellular milieu, or to changes of membrane potential. This approach gives access to valuable information on the behavior of kidney cells under extreme situations, or under the influence of drugs affecting the ion pumps or channels. 


\section{Illumination of the cells does not affect the cyst size during the first 4 hours.}

In order to test whether illumination of the cells by the excitation light used by spinning disc microscope had any significant effect on the cell or cyst volumes, the cell cysts were illuminated overnight in culture medium containing serum (Figs 1 and 2). The size of the cell cysts and lumina remained constant for 10 hours. When the culture medium was replaced with Hanks' balanced salt solution (HBSS), where glucose is the only source of energy, the cysts collapsed after four hours, presumably due to shortage of ATP and serum (Fig 2). Therefore, in the experiments aiming to analyze the cell responses to various stimuli, quantitative measurements were only carried out using images collected during the first 2.5 hours of culture under the test conditions.

\section{Lack of monovalent cations in the basal fluid induces water secretion to lumen.}

We investigated the effect of monovalent cation and chloride ion depletion in the extracellular (basal side) buffer surrounding the cysts on the cell and lumen volumes. Monovalent cations were removed from the extracellular buffer by replacing the medium with tetramethyl ammonium chloride (TMACl) buffer, which induces a hyperpolarization of the cells due to efflux of monovalent cations, and particularly potassium ions to the basal side (Vääräniemi et al. 1997). Under these conditions, an average luminal volume expansion of 2.2-fold, accompanied by a $70 \%$ increase of the apical membrane surface area, was observed within 2.5 hours (Figs 3, 4, and 7, Table 2). The cell volume increased by $24 \%$, causing a $22 \%$ increase of basal surface area. Thus the hyperpolarization clearly induced an extensive flow of water into the cells through the basal side that the apical channels were not able to secrete into the lumen at the same rate. However, the cell height remained almost constant (Table 2), suggesting that the increase of the apical membrane area resulted from the opening of the apical membrane folds, rather than from a reduction in the length of the lateral walls or changes in cell shape.

\section{Chloride ion depletion in the basal fluid induces water re-absorption.}

The effect of chloride ion depletion on the cyst and cell behavior was investigated by incubating the cultures in sodium gluconate solution. Under these conditions, the volume of the cells remained constant, 
while the lumen size decreased significantly within 2.5 hours (Figs 6 and 7, Table 2). The results show that transcellular water flow takes place from the lumen to the basal side, most likely induced by the transport of chloride ions along their concentration gradient. In order to confirm that water flow from the lumen was not due to the opening of tight junctions (TJ), we performed an immunofluorescence microscopy analysis of tight junction protein occludin and the adherens junction protein E-cadherin in the cell cysts fixed in various media (Fig 5). The TJs remained intact both in TMACl and in sodium gluconate solution, confirming that transepithelial water flow can occur in both directions under these conditions. EGTA treatment induced the clustering of occludin at the apical membranes, whereas E-cadherin staining remained localized in the lateral cell walls (Fig. 5D).

\section{Depolarizing conditions induce cell swelling and water re-absorption.}

The effects of sodium ion deficiency on cyst behavior were investigated by incubating the cells in $\mathrm{KCl}$ solution, with or without nigericin, a proton-potassium exchanger which opens the membrane to monovalent cations. Extracellular $\mathrm{KCl}$ buffer reduces the transmembrane potential of the cells, while nigericin supplement leads to a complete depolarization of the cell interior (Vääräniemi et al. 1997). In KCl buffer, without nigericin, the cell size remained constant, while lumen size decreased (Figs 6 and 7). Hence, the lack of sodium does not create a similar driving force for chloride secretion or water transport as the lack of both potassium and sodium ions did, but rather causes the re-absorption of water. Nigericin treatment in $\mathrm{KCl}$ buffer led to decreased lumen size and increased cyst size due to cell swelling by the Donnan effect, i.e. water influx caused by the presence of large inorganic anions in the cytoplasm (Figs 6 and 7, Table 2). The lumen size decreased confirming the fact that transepithelial water flow towards the lumen requires a luminal negative potential, which activates chloride channels.

\section{Agonists and inhibitors of chloride channels induce changes in the cell, cyst and lumen size.}

In order to identify possible candidate channels responsible for the chloride secretion and re-absorption, we examined the effects of known chloride channel agonists and inhibitors on the cell, cyst and lumen sizes. 
First, we tested whether the effects of forskolin and lubiprostone, two activators of chloride secretion in epithelia, could be observed in our experimental set-up. Forskolin is a cAMP agonist while lubiprostone is an activator of CFTR and ClC-2. Both reagents induced lumen expansion (Table 2 and Fig. 3), forskolin treatment led to a 1.2 -fold increase of the luminal surface area, and a 1.3-fold increase of the luminal volume, while the increases induced by lubiprostone were 1.3-fold and 1.5-fold, respectively.

We then tested the effects of two chloride channel inhibitors, CFTR $_{\text {inh }}-172$ and ANO1-inhibitor. CFTR inh -172 , which inhibits CFTR and VSORCC, completely abolished TMACl-induced lumen expansion in type II MDCK cells, while the ANO1-inhibitor only partially inhibited the lumen expansion (Fig 3, Table 2). To elucidate the mutual effects of cAMP and of the luminal negative potential, we tested the respective effects of forskolin and TMACl on cyst and luminal expansion (Table 2). Preincubation of the cell cysts with forskolin completely inhibited the TMACl-induced luminal expansion. Hence, activation of chloride channels by forskolin and TMACl are exclusive, suggesting that cAMP activation is also involved in chloride secretion in type II MDCK cells.

In order to analyze whether the activation of chloride channels could take place under depolarizing conditions, we tested the effect of lubiprostone in the presence of nigericin in $\mathrm{KCl}$ buffer. Under these conditions, lubiprostone induced increases of 1.3 -fold and 1.5 -fold in the lumen area and volume, respectively (Fig 6, Table 2). Hence, the TMACl-induced hyperpolarization and lubiprostone- or forskolininduced cAMP activation both lead to the same outcomes: increased chloride ion secretion and subsequent transepithelial water flow, resulting in lumen expansion due to intraluminal hydrostatic pressure.

The time-course of the changes in the cell, cyst and lumen volumes under the different conditions is summarized in Fig. 7.

\section{Discussion}

Three-dimensional cell culture systems can be of utmost importance for testing therapeutic applications, e.g. in testing the therapeutic response of tumour cells to various compounds or irradiation, or in evaluating the invasive capacity of transformed cells in vitro culture models can be expected to have 
significant potential in designing preclinical tests and models for genome editing. One such example is provided by the work by Schwank and co-workers (2013), who were able to show that human intestinal stem cells expressing normal CFTR could be stimulated by CAMP, inducing a rapid swelling of the organoid cluster. This response was lost in organoids derived from cystic fibrosis patients and could be rescued by functional repair of CFTR by genome editing.

Very little data is available relating to the actual behavior of a three-dimensional epithelial cyst in response to extracellular stimuli, such as changes in membrane potential or extracellular ion composition. In their pioneering work, Tanner and co-workers (1992) showed that MDCK cells cultured in a three-dimensional collagen matrix were capable of rapid responses to changes of the osmotic equilibrium of the environment. When MDCK cysts were exposed to hypotonic medium, the cells first swelled, and this was followed by luminal expansion. The phenomena were reversible and both the lumen and cell volumes returned to their original states when the cultures were returned to the normal medium. Hence, the MDCK cells were shown to be capable of transcellular water flow. Several works have since shown that cAMP stimulation by forskolin activates apical chloride channels and induces transepithelial water flow into the MDCK lumen (Buchholz et al. 2014a; Li et al. 2004; Tanner et al. 1992; Yuajit et al. 2013).

In the present study, we used fluorescent markers and high-resolution spinning disc confocal microscope to collect three-dimensional image stacks of the cysts. Using the 3D data we were able to calculate the cyst, cell and lumen volumes separately. The short durations of the experiments allowed us to eliminate the influence of changing cell numbers on the interpretation of the result. We chose to use type II MDCK cells in this model, since they form well-developed cysts in 3D culture, thereby facilitating the measurements of both the cell and luminal dimensions. These cells are presumed to originate from the proximal tubule of the canine kidney; the chloride channels expressed by these cells are not well characterized, although it has been shown that they lack CFTR (Mohamed et al. 1997). Our results show that the effects of depolarization, hyperpolarization, and several agonists and inhibitors of chloride channels can be studied using the type II MDCK cell model and high resolution spinning disc confocal microscopy. 
In normal circumstances, the characteristic feature of mammalian kidney function is glomerular filtration and subsequent reabsorption of sodium and chloride ions in renal tubules, as well as water in different segments of nephrons, in a highly regulated manner. It has been suggested, however, that transepithelial salt and fluid secretion mechanisms allow mammalian renal tubules to finely regulate extracellular fluid volume and composition, and to maintain urine formation during the cessation of glomerular filtration (Grantham and Wallace 2002). The dominance of glomerular filtration also appears to vary between the animals living in a freshwater aquatic environment or in a desert environment (Grantham and Wallace 2002).

The secretory properties of proximal and distal tubule are important in acute or chronic renal failure and in hypertonic dehydration. In situations where glomerular filtration is reduced or interrupted, the task of mammalian proximal tubular cells is to secrete hippurate and other similar substances, in order to keep the tubule lumina open and ensure the elimination of toxic incredients to urine. Here we have shown that chloride ions and water can flow through type II MDCK epithelial cells in both directions depending on the ion composition of the basal fluid and the potential difference across the cell membranes. A luminal negative potential induces water flow towards the lumen, while depolarization lead to the re-absorption of water from the lumen and its flow towards the basal side. We have also shown that, on its own, a low sodium ion concentration is not critical, whereas a lack of both potassium and sodium triggered extensive water inflow from basal side. The present method also allows the investigation of the mutual roles of transporters located at the basal membrane, such as passive potassium channels, sodium-potassium ATPase, sodium-potassium-chloride cotransporters, and various aquaporins, in response to specific inhibitors to these proteins. The work presented here focuses on the transport capacity of MDCK cysts and the electrophysiological regulation of water transport.

Our results suggest that forskolin and lubiprostone are able to activate the apical secretory pathways of type II MDCK cells. The effects may be transmitted via the activation of apical chloride channels, or through other cAMP-mediated mechanisms. In this context, it is interesting that both forskolin and CPT-cAMP have 
been shown to induce the translocation of a water channel aquaporin 2 to the apical membrane of MDCK cell cysts (Rice et al. 2015). Lubiprostone has been shown to have multimodal prostaglandin-like effects in the intestine and it enhances the contractility of intestinal smooth muscles (Chan and Mashimo 2013). In our experimental set-up TMACl-treatment induced cell swelling, suggesting that volume-sensitive channels may also participate in chloride secretion. Li et al. (2004) have shown that CFTR inhibitors diminish the forskolin-stimulated enlargement of type I MDCK cysts, whereas inhibitors both to CFTR and ANO1 caused a reduction of cAMP-stimulated $\mathrm{Cl}^{-}$ion current. Moreover, in their more recent studies Li et al. (2012) showed that type I MDCK cells expressing the F508del-CFTR formed very few, tiny cysts. Hence, type I and type II MDCK cells have different requirements for their differentiation and type II MDCK cells form welldeveloped cysts without expression of CFTR. In our hands, ANO1 inhibitor could not prevent the lumen expansion caused by TMACl; the channel may not even be expressed in type II MDCK cells. It seems that the calcium-activated chloride secretion is dominant under hypoxic conditions where CFTR expression is suppressed (Buchholz et al. 2014b). In fact, ANO1 is more abundant in acinar cells, such as salivary glands, whereas CFTR is a key player in secretion in ductal cells, from which the type I MDCK cells originate (Hong et al. 2014).

In conclusion, type II MDCK cells are capable of both absorption and secretion of chloride ions and water, depending on the ion composition of the basal extracellular fluid. A lack of monovalent cations induces transepithelial water flow towards the lumen, with a subsequent lumen expansion via cAMP-mediated signalling pathway and/or through the activation of voltage- and volume-sensitive chloride channels. A lack of chloride ions, in turn, induces the reabsorption of water from the lumen. Depolarizing conditions also induces water reabsorption, which can be reversed by the direct activation of chloride and water secretion using lubiprostone. This flexibility shows how tissue homeostasis can be maintained in severe circumstances, such as the failure of glomerular filtration, hypertonic dehydration, or external haemorrhage. 


\section{Disclosure}

The authors declare no competing interests.

\section{Acknowledgments}

The authors would like to thank Dr. Reika Watanabe and Dr. Guillaume Castillon, Department of Biochemistry, University of Geneve, Switzerland for providing the Venus-CD59 MDCK cells. Also, special thanks to Dr. Veli-Pekka Ronkainen for assistance with microscopes, Civ. Eng. Antti Viklund for help with image analysis and the use of Imaris and M.Sc. Lisette Van Tassel for help with statistics and Dr. Anthony Heape for language revision.

\section{References}

Bi, M., Hong, S., Zhou, H., Wang, H., Wang, L., and Zheng, Y. 2013. Chloride channelopathies of ClC-2. Int. J. Mol. Sci. 27: 218-49.

Bijvelds, M., Bot, A., Escher, J., and De Jonge, H. 2009. Activation of intestinal Cl- secretion by lubiprostone requires the cystic fibrosis transmembrane conductance regulator. Gastroenterology, 137(3):976-85.

Buchholz, B., Faria, D., Schley, G., Schreiber, R., Eckardt, K., and Kunzelmann, K. 2014a. Anoctamin 1 induces calcium-activated chloride secretion and proliferation of renal cyst-forming epithelial cells. Kidney Int. 85(5): 1058-1067.

Buchholz, B., Schley, G., Faria, D., Kroening, S., Willam, C., Schreiber, R. et al. 2014b. Hypoxia-inducible factor-1 $\alpha$ causes renal cyst expansion through calcium-activated chloride secretion. J. Am. Soc. Nephrol. 25(3): 465-474. 
Castillon, G., Michon, L., and Watanabe, R. 2013. Apical sorting of lysoGPI-anchored proteins occurs independent of association with detergent-resistant membranes but dependent on their $\mathrm{N}$ glycosylation. Mol. Biol. Cell. 24(12): 2021-2033.

Chan, W., and Mashimo, H. 2013. Lubiprostone increases small intestinal smooth muscle contractions through a prostaglandin E receptor 1 (EP1)-mediated pathway. J. Neurogastroenterol. Motil. 19(3): 312-

8.

Cuppoletti, J., Malinowska, D., Tewari, K., Li, Q., Sherry, A., Patchen, M., and Ueno, R. 2004. SPI-0211 activates T84 cell chloride transport and recombinant human CIC-2 chloride currents. Am. J. Physiol. 287(5): C1173-83.

Datta, A., Bryant, D., and Mostov, K. 2011. Molecular regulation of lumen morphogenesis. Curr. Biol. 21(3): R126-136.

Debnath, J., Muthuswamy, S., and Brugge, J. 2003. Morphogenesis and oncogenesis of MCF-10A mammary epithelial acini grown in three-dimensional basement membrane cultures. Methods, 30(3): 256-268.

Dukes, J., Whitley, P., and Chalmers, A. 2011. The MDCK variety pack: choosing the right strain. BMC Cell Biol. 7: 12-43.

Frizzell, R., and Hanrahan, J. 2012. Physiology of epithelial chloride and fluid secretion. Cold. Spring. Harb. Perspect. Med. 2(6): a009563.

Grantham, J., and Wallace, D. 2002. Return of the secretory kidney. Am. J. Physiol. 282(1): F1-9.

Hoffmann, E., Holm, N., and Lambert, I. 2014. Functions of volume-sensitive and calcium-activated chloride channels. IUBMB Life, 66(4):257-67.

Hong, J., Park, S., Shcheynikov, N., and Muallem, S. 2014. Mechanism and synergism in epithelial fluid and electrolyte secretion. Pflugers Arch. 466(8): 1487-99. 
Kunzelmann, K. 2015. TMEM16, LRRC8A, bestrophin: chloride channels controlled by $\mathrm{Ca}(2+)$ and cell volume. Trends Biochem. Sci. 40(9): 535-43.

Li, H., Findlay, I., and Sheppard, D.N. 2004. The relationship between cell proliferation, $\mathrm{Cl}$ - secretion, and renal cyst growth: a study using CFTR inhibitors. Kidney Int. 66(5): 1926-1938.

Li, H., Yang, W., Mendes, F., Amaral, M., and Sheppard, D. 2012. Impact of the cystic fibrosis mutation F508del-CFTR on renal cyst formation and growth. Am. J. Physiol. 303(8): F1176-F1186.

Melis, N., Tauc, M., Cougnon, M., Bendahhou, S., Giuliano, S., Rubera, I., and Duranton, C. 2014. Revisiting CFTR inhibition: a comparative study of CFTRinh -172 and GlyH-101 inhibitors. Br. J. Pharmacol. 171(15): 3716-27.

Mohamed, A., Ferguson, D., Seibert, F., Cai, H., Kartner, N., Grinstein, S., Riordan, J., and Lukacs, G. 1997. Functional expression and apical localization of the cystic fibrosis transmembrane conductance regulator in MDCK I cells. Biochem. J. 15: 259-65.

Namkung, W., Phuan, P., and Verkman, A. 2011. TMEM16A inhibitors reveal TMEM16A as a minor component of calcium-activated chloride channel conductance in airway and intestinal epithelial cells. J. Biol. Chem. 21(3): 2365-74.

Noda, Y., Sohara, E., Ohta, E., and Sasaki, S. 2010. Aquaporins in kidney pathophysiology. Nat. Rev. Nephrol. 6(3): 168-178.

Rice, W., Li, W., Mamuya, F., McKee, M., Păunescu, T., and Lu, H. 2015. Polarized trafficking of AQP2 revealed in three dimensional epithelial culture. PLoS One, 10(7):e0131719

Rivier, A., Castillon, G., Michon, L., Fukasawa, M., Romanova-Michaelides, M., Jaensch, N., et al. 2010. Exit of GPI-anchored proteins from the ER differs in yeast and mammalian cells. Traffic, 11(8): 10171033. 
Rodriguez-Boulan, E., and Macara, I. 2014. Organization and execution of the epithelial polarity programme. Nat. Rev. Mol. Cell. Biol. 15(4): 225-242.

Schwank, G., Koo, B., Sasselli, V., Dekkers, J., Heo, I., Demircan, T. et al. 2013. Functional repair of CFTR by CRISPR/Cas9 in intestinal stem cell organoids of cystic fibrosis patients. Cell Stem Cell, 13(6): 653658.

Sullivan, L., Wallace, D., and Grantham, J. 1998. Chloride and fluid secretion in polycystic kidney disease. J. Am. Soc. Nephrol. 9(5): 903-916.

Tanner, G., Maxwell, M., and McAteer, J. 1992. Fluid transport in a cultured cell model of kidney epithelial cyst enlargement. J. Am. Soc. Nephrol. 2(7): 1208-1218.

Terryn, S., Ho, A., Beauwens, R., and Devuyst, O. 2011. Fluid transport and cystogenesis in autosomal dominant polycystic kidney disease. Biochim. Biophys. Acta, 1812(10): 1314-1321.

Töyli, M., Rosberg-Kulha, L., Capra, J., Vuoristo, J., and Eskelinen, S. 2010. Different responses in transformation of MDCK cells in 2D and 3D culture by v-Src as revealed by microarray techniques, RTPCR and functional assays. Lab. Invest. 90(6): 915-928.

Vääräniemi, J., Huotari, V., Lehto, V., and Eskelinen, S. 1994. The effects of PMA and TFP and alterations in intracellular $\mathrm{pH}$ and calcium concentration on the membrane associations of phospholipid-binding proteins fodrin, protein kinase C and annexin II in cultured MDCK cells. Biochim. Biophys. Acta, 1189(1): 21-30.

Vääräniemi, J., Huotari, V., Lehto, V., and Eskelinen S. 1997. Effect of PMA on the integrity of the membrane skeleton and morphology of epithelial MDCK cells is dependent on the activity of amiloridesensitive ion transporters and membrane potential. Eur. J. Cell. Biol. 74(3): 262-272.

Verkman, A., Anderson, M., and Papadopoulos, M. 2014. Aquaporins: important but elusive drug targets. Nat. Rev. Drug Discov. 13(4): 259-277. 
Wallace, D. 2011. Cyclic AMP-mediated cyst expansion. Biochim. Biophys. Acta, 1812(10): 1291-1300.

Yagi, S., Matsuda, M., and Kiyokawa, E. 2012. Suppression of Rac1 activity at the apical membrane of MDCK cells is essential for cyst structure maintenance. EMBO Rep. 13(3): S3-4.

Yang, B., Sonawane, N., Zhao, D., Somlo, S., and Verkman, A. 2008. Small-molecule CFTR inhibitors slow cyst growth in polycystic kidney disease. J. Am. Soc. Nephrol. 19(7): 1300-1310.

Yuajit, C., Homvisasevongsa, S., Chatsudthipong, L., Soodvilai, S., Muanprasat, C., and Chatsudthipong, V. 2013. Steviol reduces MDCK Cyst formation and growth by inhibiting CFTR channel activity and promoting proteasome-mediated CFTR degradation. PLoS One, 8(3): e58871. 
Table 1. The ion compositions (in $\mathrm{mM}$ ) of the buffers used in time-lapse imaging of MDCK cysts. All media contained $20 \mathrm{mM}$ Hepes and $18 \mathrm{mM}$ glucose. $\mathrm{pH}$ was adjusted to 7.4 with phosphate salts, $\mathrm{KOH}, \mathrm{TMAOH}$ or $\mathrm{NaOH}$, depending on the main cation in the buffer.

\begin{tabular}{|l|cccccc|}
\hline Solution & $\mathrm{Na}^{+}$ & $\mathrm{K}^{+}$ & $\mathrm{Ca}^{2+}$ & $\mathrm{Cl}^{-}$ & $\mathrm{TMA}^{+}$ & gluconate \\
\hline $\mathrm{HBSS}$ & 141.4 & 5.8 & 1.25 & 144.7 & - & - \\
TMACl & - & 5 & 1 & 136 & 130 & - \\
Na-gluconate & 140.5 & - & 5.7 & 15.4 & - & 140 \\
$\mathrm{KCl}$ & - & 131.5 & 1 & 132 & - & - \\
\hline
\end{tabular}

Table 2. Changes in cell, cyst and lumen volumes, apical and basal membrane areas and cell height after 2.5 hours in various environments.

\begin{tabular}{|l|cccccc|}
\hline Condition & $\frac{V_{\text {final }}}{V_{\text {init }}}$ cell & $\frac{V_{\text {final }}}{V_{\text {init }}}$ lumen & $\frac{V_{\text {final }}}{V_{\text {init }}}$ cyst & $\frac{A_{\text {final }}}{A_{\text {init }}}$ basal & $\frac{A_{\text {final }}}{A_{\text {init }}}$ apical & $\frac{h_{\text {final }}}{h_{\text {init }}}$ \\
\hline Ctrl (medium) & 1.04 & 0.96 & 1.02 & 1.01 & 0.97 & N/A \\
Ctrl (HBSS) & 1.06 & 1.10 & 1.06 & 1.04 & 1.06 & 1.00 \\
Forskolin in HBSS & 0.84 & 1.32 & 0.93 & 0.95 & 1.20 & N/A \\
TMACl & 1.24 & 2.22 & 1.34 & 1.22 & 1.70 & 0.93 \\
TMACl + CFTR inh-172 & 1.01 & 0.82 & 0.99 & 0.99 & 0.88 & N/A \\
TMACl + CaCC inh-A01 & 0.93 & 1.37 & 0.96 & 0.97 & 1.24 & N/A \\
Lubiprostone in HBSS & 1.09 & 1.52 & 1.14 & 1.09 & 1.32 & N/A \\
Lubiprostone in KCl- & 1.15 & 1.48 & 1.21 & 1.14 & 1.30 & N/A \\
nigericin & & & & & & N/A \\
Forskolin + TMACl & 1.14 & 0.96 & 1.10 & 1.07 & 0.97 & N/A \\
Na-gluconate & 1.08 & 0.58 & 0.98 & 0.99 & 0.69 & N/A \\
KCl & 1.02 & 0.89 & 1.01 & 1.00 & 0.92 & N/A \\
KCl + nigericin & 1.34 & 0.89 & 1.29 & 1.18 & 0.93 & N \\
\hline
\end{tabular}


Legends to Figures

Fig 1. Visualization of Venus-CD59 MDCK cells in culture medium and 3D rendering of the luminal space with Imaris software. In order to determine the shape of the lumen within the cyst, a threedimensional model of the cyst was generated with the aid of Imaris software. Confocal sections of 5 day cysts were taken, with green being the lumen and blue the lateral membranes ( $A$ and $B$ ). Subsequently, a 3D illustration of a lumen ( $C$ and $D$, from $A$ and $B$, respectively) was rendered with the Imaris software. The generated model demonstrates that the lumina are also spherical. This allowed us to treat the cyst and its lumen as a sphere, which in turn allowed us to use maximal cross-section areas for later calculations of cell, cyst and lumen volumes and surface areas. The scale bar represents $20 \mu \mathrm{m}$.

Fig 2. Visualization of Venus-CD59 MDCK cysts in culture medium and HBSS. Venus-CD59 MDCK cysts were grown in Matrigel in the cell culture incubator up to the desired point, TMA-DPH membrane dye was added and a Z-stack was collected with the Spinning Disc microscope, at 30 minute intervals. When the cysts were cultivated in the presence of medium supplemented with FBS (A), cysts were able to survive and even increase in size for 30 hours, after which they started to collapse due to the laserinduced phototoxicity. Next the cells were visualized in HBSS with glucose as a source of energy. The maximal cross-section areas of the lumen and cyst were measured and the mean values with standard error as a function of time are shown in B. The cyst size remained constant for 4 hours before collapsing, showing that HBSS alone does not trigger water influx into the cells. The scale bar represents $20 \mu \mathrm{m}$.

Fig 3. The summary of the changes in both the lumen and cyst areas in the presence of chloride channel activators and inhibitors and in various hyperpolarizing environments. Relative changes of lumen (A) and cyst (B) maximal cross-section areas at different time points in different conditions are shown as a histogram. Standard errors for different time points were calculated from original ratios.

Two-tailed Student's paired t-test with equal variance was used to assess the significance of the changes of lumen and cyst areas with time. $P$-values were categorized as not significant $(P>0.05)$, 
significant $\left(P<0.05\right.$, denoted with $\left.{ }^{*}\right)$ and very significant $\left(P<0.01\right.$, denoted with $\left.{ }^{* *}\right)$. In the presence of $\mathrm{TMACl}$, a remarkable growth in the luminal area was measured. The cyst area underwent significant growth in the presence of TMACl due to the enormous growth of the lumen. This luminal expansion did not occur when chloride channel inhibitor $\mathrm{CFTR}_{\text {inh }}-172$ was supplemented with TMACl. $\mathrm{CaCC}_{\text {inh }}-\mathrm{A01}$ reduced the lumen expansion, but to a lesser extent than CFTR $_{\text {inh }}-172$. The CFTR and CIC-2 activator, lubiprostone, and the cAMP agonist, forskolin, both induced lumen expansion in HBSS. No significant changes were observed in HBSS without supplements.

Fig 4. Visualization of Venus-CD59 MDCK cysts in the presence of tetramethylammonium chloride (TMACI). A distinct expansion of the lumen can already be seen after 1 hour in TMACl. The scale bars represent $20 \mu \mathrm{m}$.

Fig 5. Immunofluorescence microscopy of the tight junction component occludin and the adherens junction component E-cadherin in cysts in the presence of HBSS, tetramethylammonium chloride (TMACl), sodium gluconate, or PBS supplemented with 1 mM EGTA. In HBSS control (A), occludin (red) forms a continuous line along the apical surface (arrows). This was undisturbed after 2 hours in TMACl ( $\mathrm{B}$, arrows) or in sodium gluconate (C, arrows). E-cadherin (green) is located along the lateral membranes in all of these conditions. EGTA-treatment caused clustering of occludin at the apical surfaces ( $D$, arrows). White squares mark an area that is shown enlarged in the inserts in the lower left corner of each image. The scale bar represents $20 \mu \mathrm{m}$.

Fig 6. The summary of the changes in both lumen and cyst areas in various depolarizing environments. Relative changes of lumen (A) and cyst (B) maximal cross-section areas at different time points in different conditions are shown as a histogram. Standard errors for different time points were calculated from original ratios.

Two tailed Student's paired t-test with equal variance was used to assess the significance of the changes of lumen and cyst areas with time. $P$-values were categorized as not significant $(P>0.05)$, 
significant $\left(P<0.05\right.$, denoted with $\left.{ }^{*}\right)$ and very significant $\left(P<0.01\right.$, denoted with $\left.{ }^{* *}\right)$. None of the depolarizing environments alone could promote lumen expansion.

Fig 7. Time-courses of changes in cell, cyst and lumen volumes in HBSS in the presence of lubiprostone $(A, E), T M A C l(B, F)$, sodium gluconate buffer $(C, G)$ and in $\mathrm{KCl}$ in the presence of nigericin $(D, H)$ are presented as normalized to values at the time point zero $(A, B, C, D)$, or as mean values of measured cysts, lumina and all cells within the cysts (in $\left.\mu m^{3}\right)(E, F, G, H)$. 

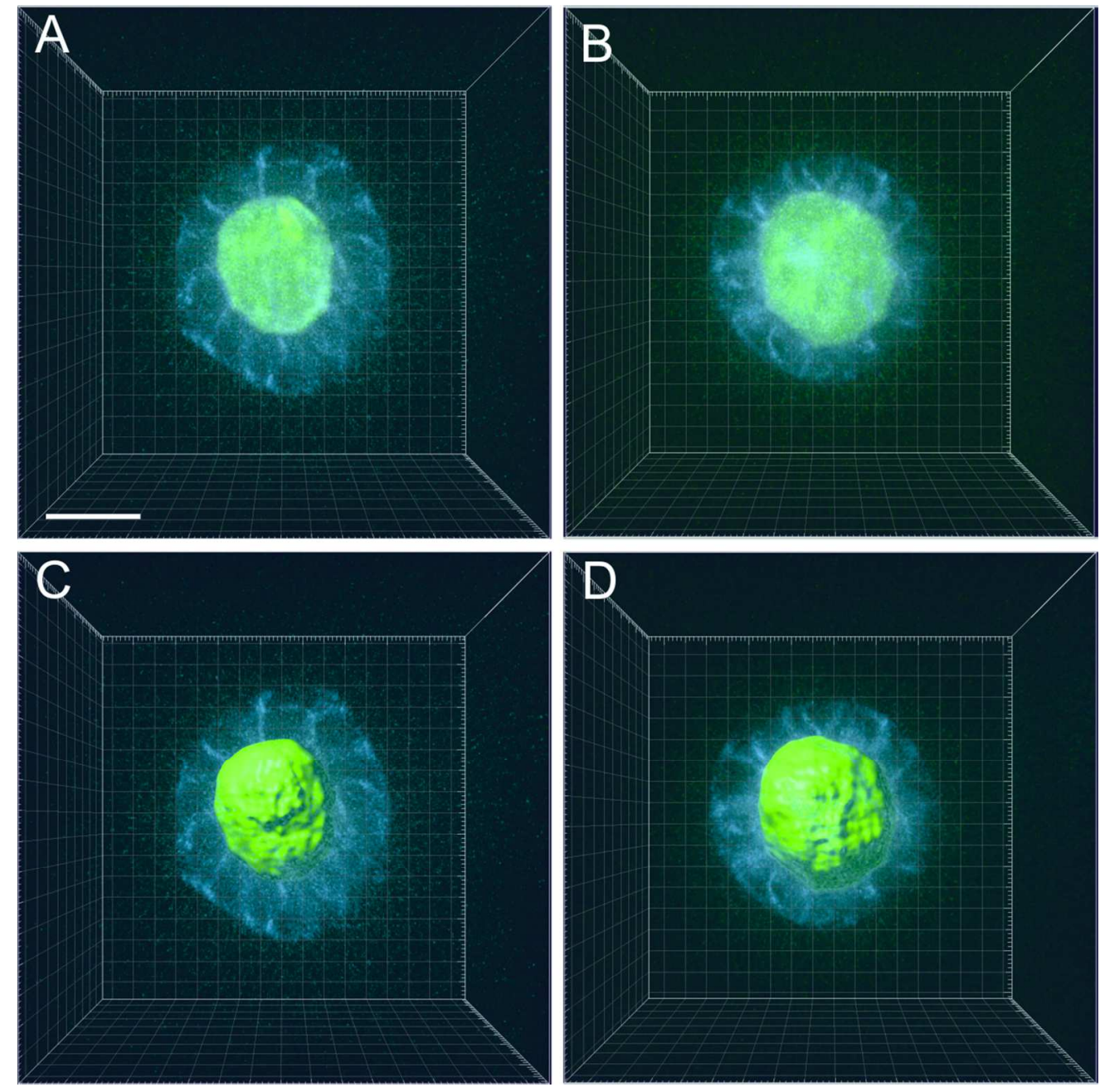

$108 \times 108 \mathrm{~mm}(300 \times 300 \mathrm{DPI})$ 

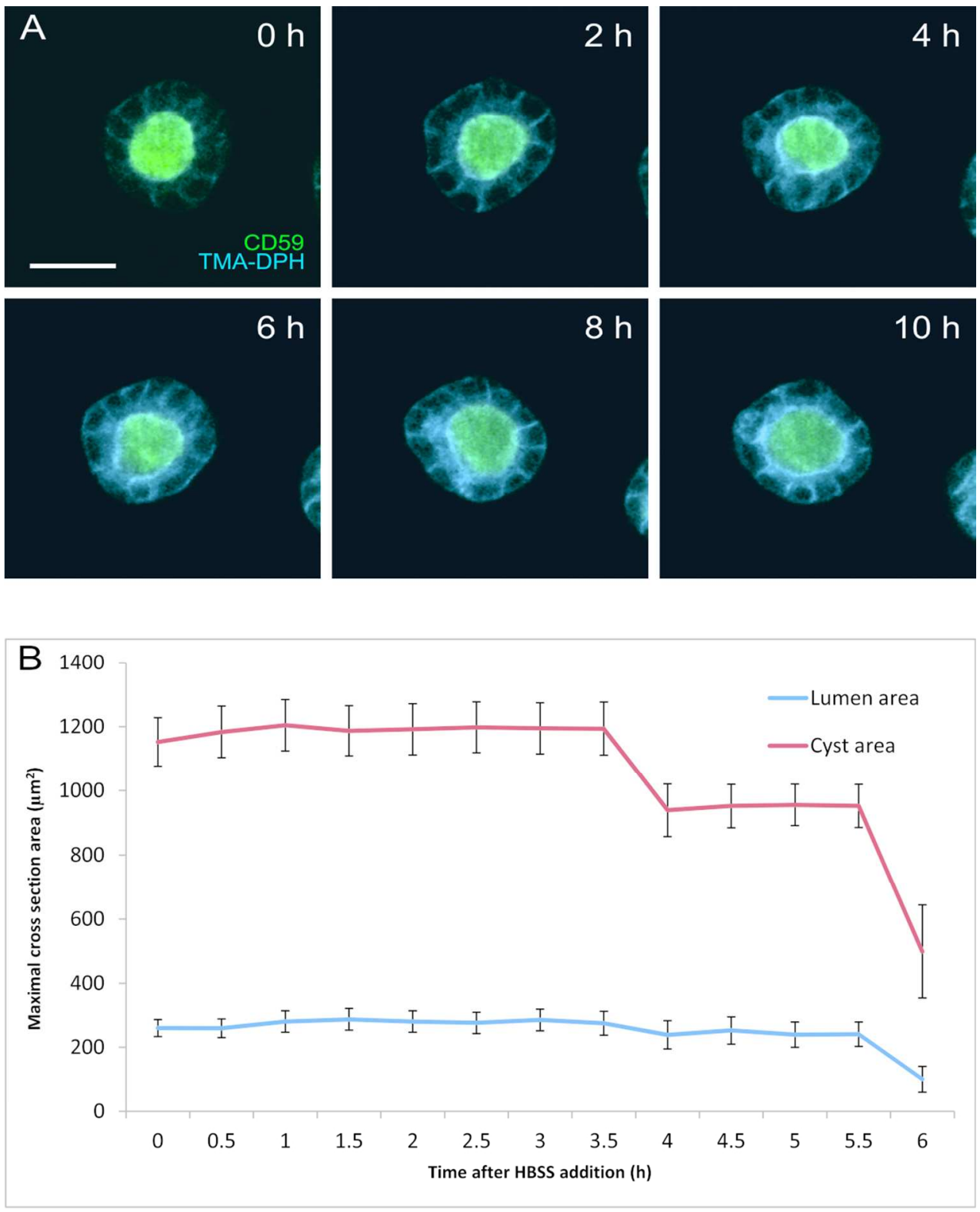

$103 \times 128 \mathrm{~mm}(300 \times 300 \mathrm{DPI})$ 

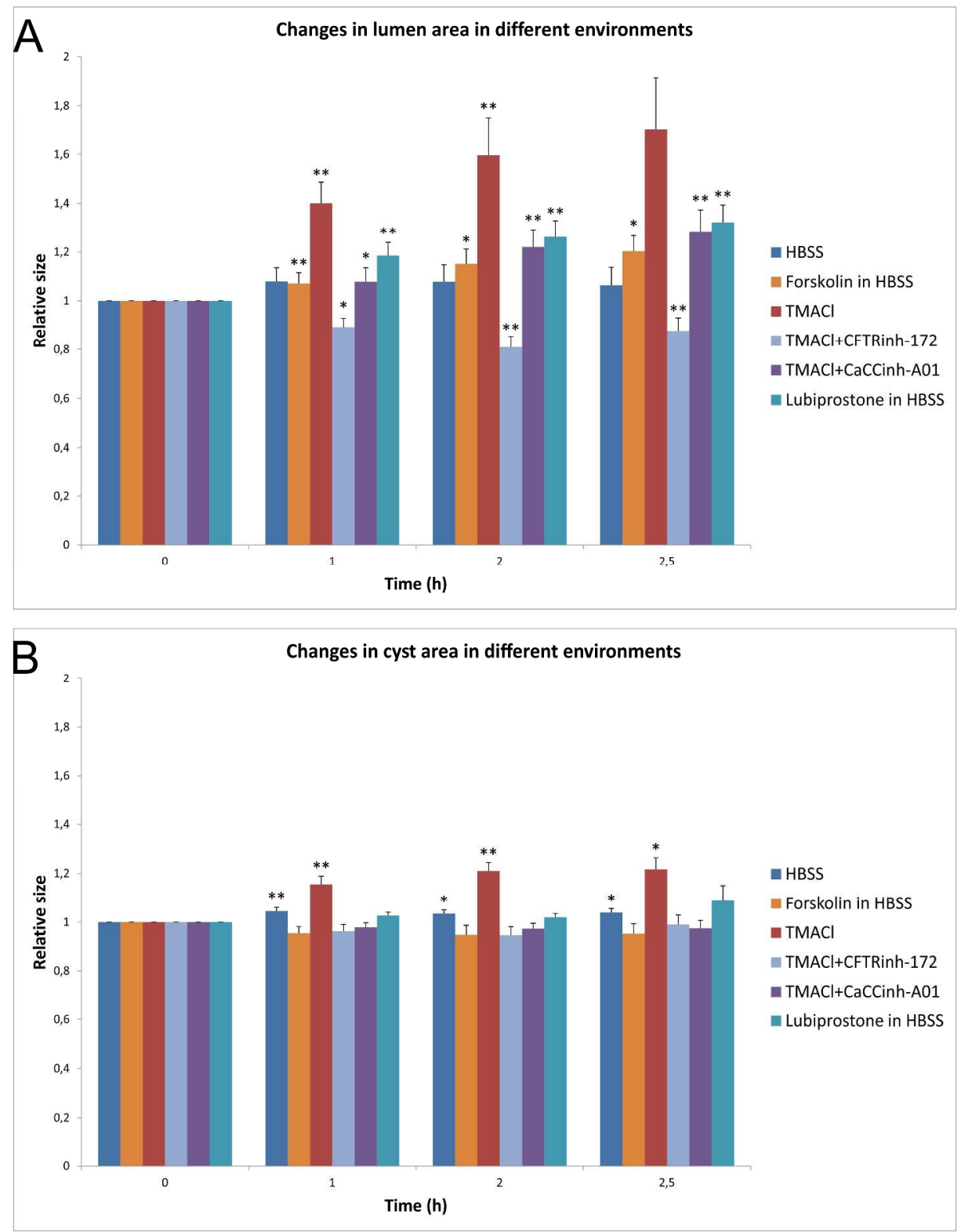

$268 \times 347 \mathrm{~mm}(300 \times 300 \mathrm{DPI})$ 


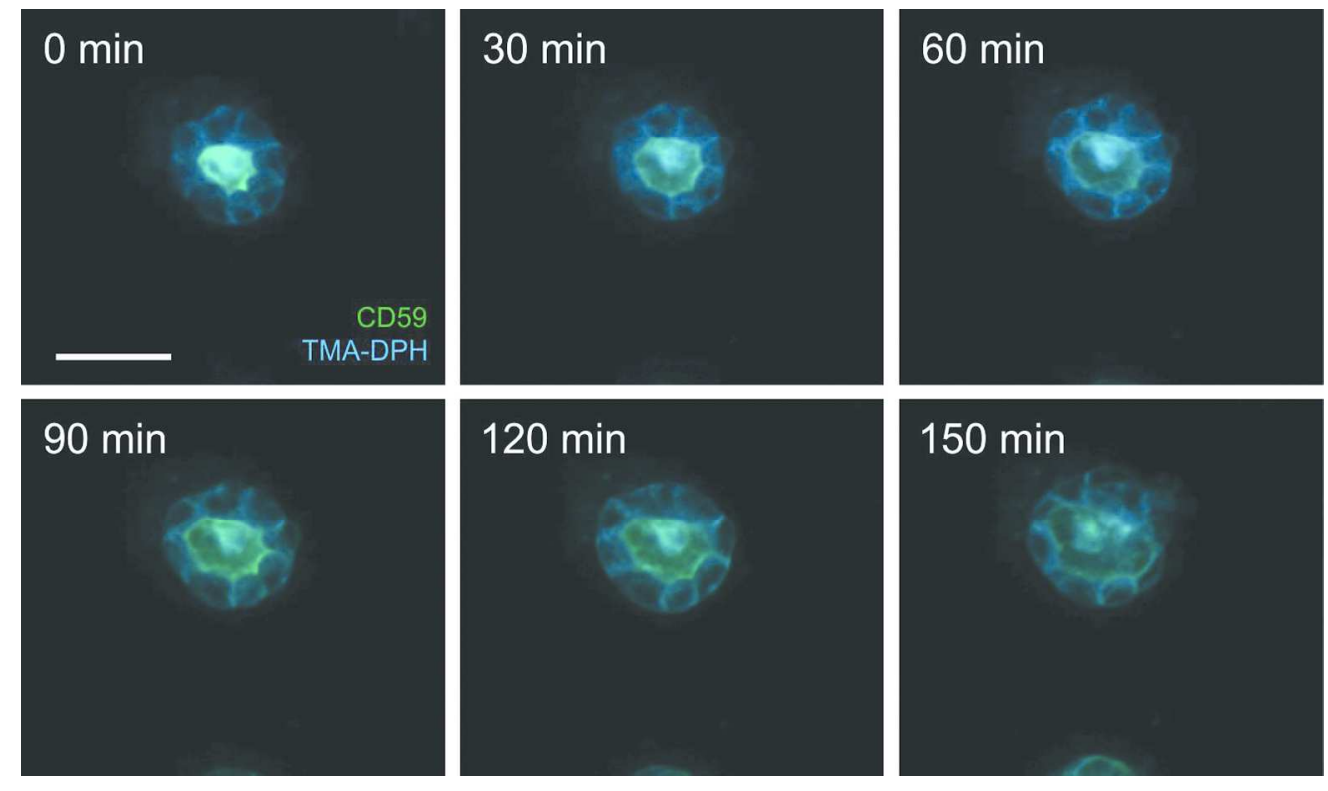

$209 \times 123 \mathrm{~mm}(300 \times 300 \mathrm{DPI})$ 

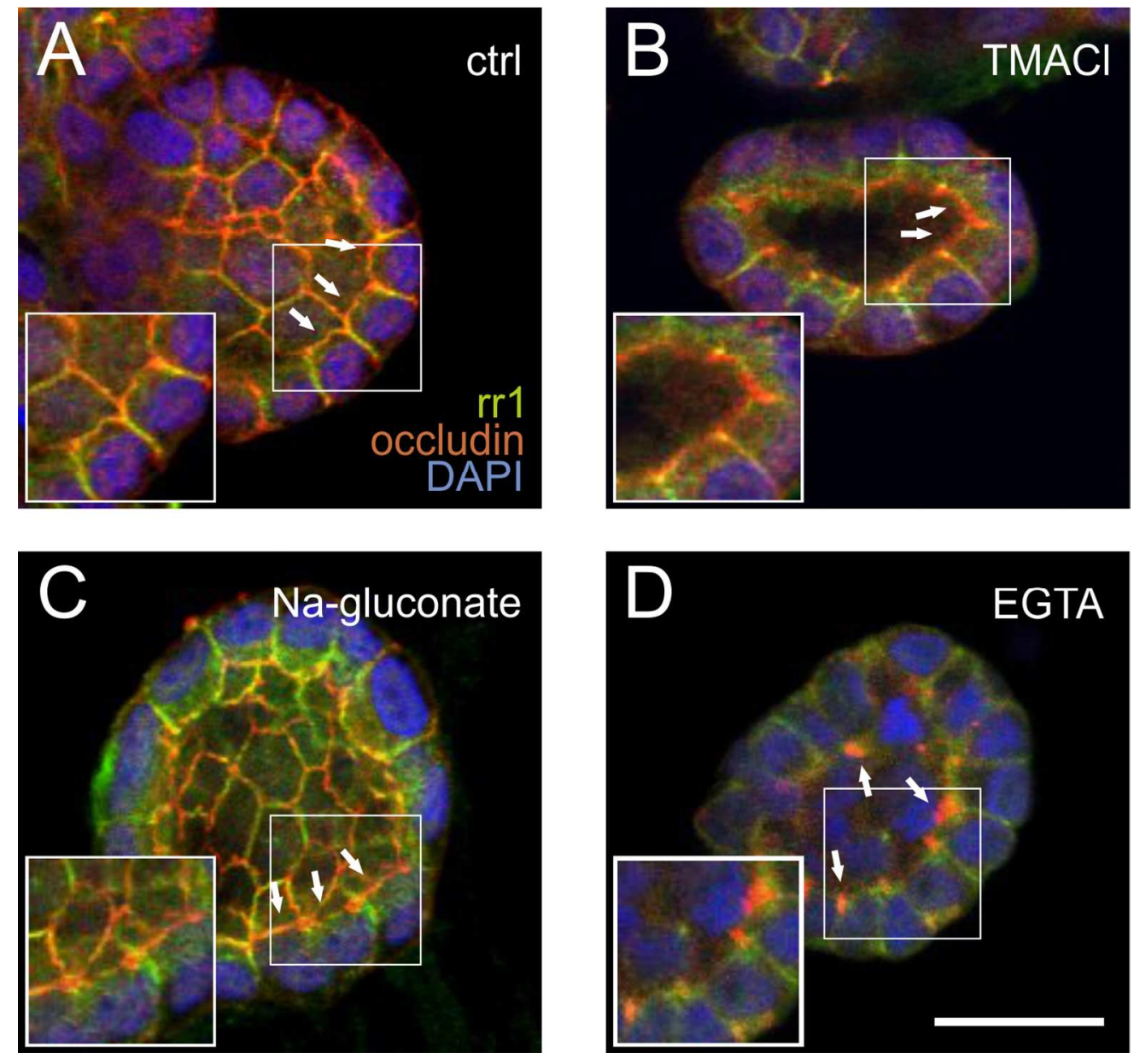

$130 \times 122 \mathrm{~mm}(300 \times 300 \mathrm{DPI})$ 

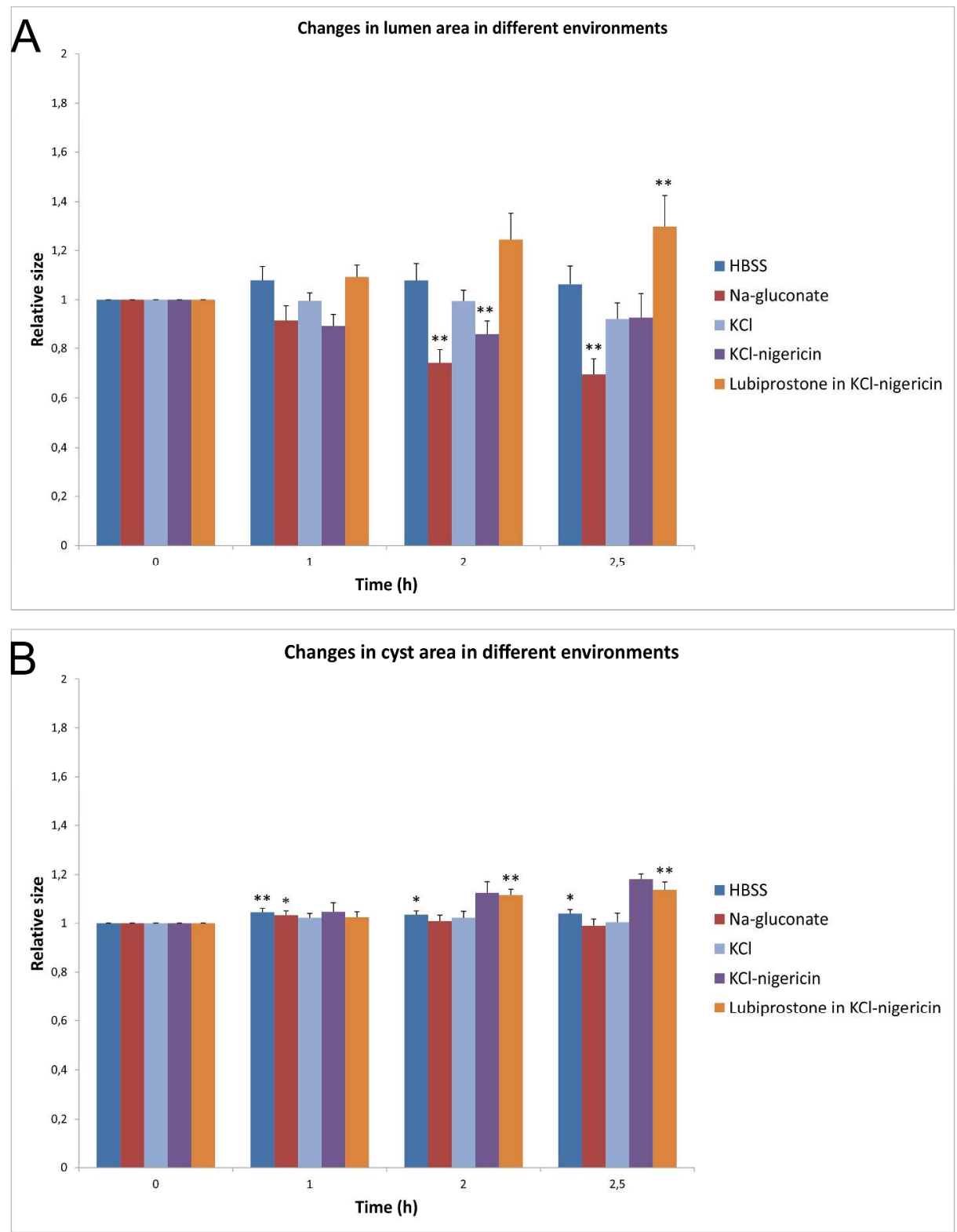

$268 \times 347 \mathrm{~mm}(300 \times 300 \mathrm{DPI})$ 

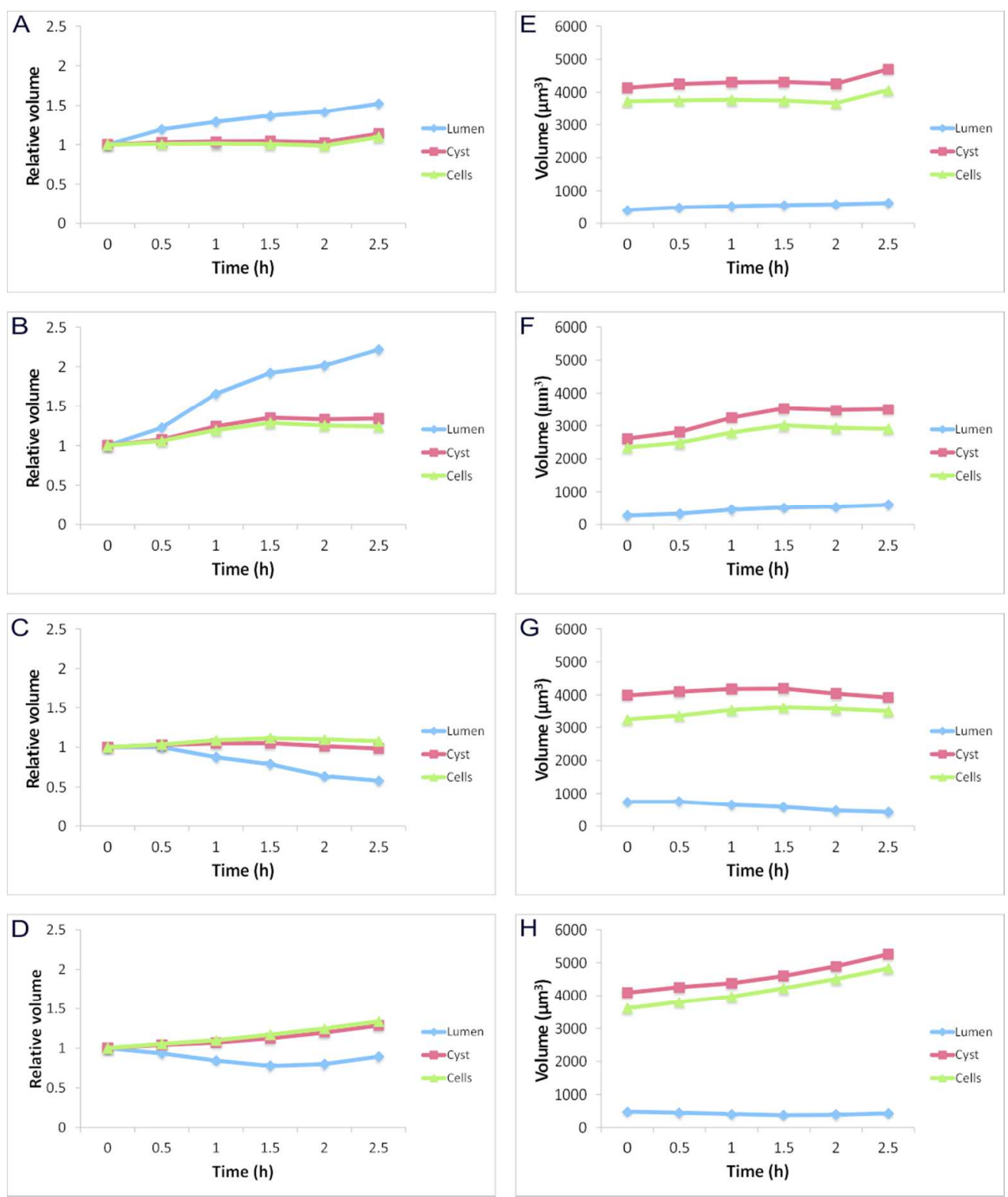

$101 \times 120 \mathrm{~mm}(300 \times 300$ DPI $)$ 